\title{
On a Conjecture concerning the Petersen Graph: Part II
}

\author{
Michael D. Plummer \\ Department of Mathematics \\ Vanderbilt University \\ Nashville, TN 37215, U.S.A. \\ michael.d.plummer@vanderbilt.edu
}

\author{
Xiaoya Zha \\ Department of Mathematical Sciences \\ Middle Tennessee State University \\ Murfreesboro, TN 37132, U.S.A. \\ xzha@mtsu.edu
}

Submitted: May 5, 2012; Accepted: Feb 13, 2014; Published: Feb 21, 2014

Mathematics Subject Classifications: 05C38, 05C40

\begin{abstract}
Robertson conjectured that the only 3-connected, internally 4-connected graph of girth 5 in which every odd cycle of length greater than 5 has a chord is the Petersen graph. We provide a counterexample to this conjecture.
\end{abstract}

\section{Introduction and Terminology}

A 3-connected graph $G$ is said to be internally 4-connected if every cutset of three vertices is the neighborhood set of a fourth vertex. In [2] it was shown, by providing a counterexample, that the conclusion of the Robertson conjecture [3] stated in the Abstract is false, if the assumption that the graph is internally 4-connected is dropped.

As also shown in [2], Robertson's Conjecture is true for many graphs; for example, for all cubic graphs and for those graphs which contain a girth cycle $C$ such that $N(C)-V(C)$ contains a path of length 2. (Here $N(C)$ denotes the neighborhood of $C$, namely, the set of all vertices adjacent to at least one vertex of $C$.) It was also proved in [2] that given any 5-cycle $C$ in a counterexample to Robertson's Conjecture, $N(C)-V(C)$ cannot be an independent set.

Thus if $G$ is any counterexample to Robertson's Conjecture, $G$ must have the following property:

(*) For every girth cycle $C$ in $G, N(C)$ consists of a matching of size at least 1, together with a set in independent vertices.

Note, however, that Robertson's conjecture is true for many graphs having property $(*)$. On the other hand, this all shows that any counterexample to Robertson's Conjecture 
must possess a rather specific structure. Indeed, the counterexample in the present paper, which is internally 4-connected, was constructed based on this structure.

We describe the construction of our counterexample in several steps due to its size and complexity. Our first building block is the Heawood graph shown in Figure 1.1.



Figure 1.1: The Heawood graph

This 3-connected internally 4-connected bipartite graph on fourteen vertices has girth 6 and has arisen in a number of graph theory settings such as vertex and edge coloring, factorization, automorphism groups and topological embedding. (Cf. [4].)

Next we connect three copies of the Heawood graph, $H_{1}, H_{2}$ and $H_{3}$, to build a larger graph which we call a Heawood triple. It is displayed in Figure 1.2. This graph is formed by joining the three copies of the Heawood graph in a circular manner where we join each of the three to its successor via a matching of size 2. (Cf. Figure 1.2.)

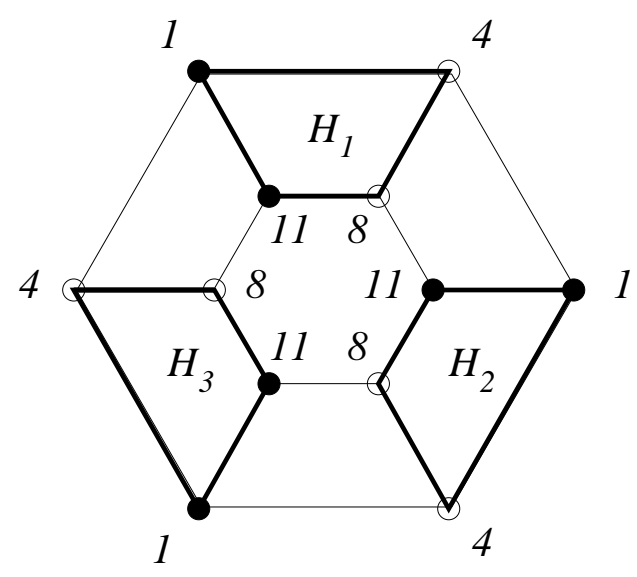

Figure 1.2: A Heawood triple

Our next building block graph is called the frame graph and will be denoted by $\mathcal{F}$. This graph is shown in Figure 1.3. 


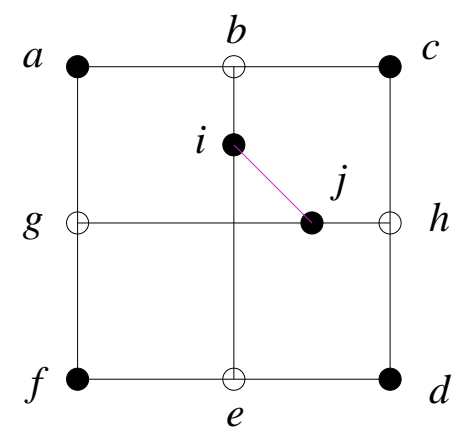

Figure 1.3: The frame graph $\mathcal{F}$

Next we attach twenty-one vertices of degree 1 to $\mathcal{F}$ as shown in Figure 1.4. The set of these vertices will be denoted by $V_{21}$.

We now add six edges from $\left\{k_{1}, k_{2}, k_{3}\right\}$ to six vertices of $V_{21}$ to obtain the graph shown in Figure 1.5.

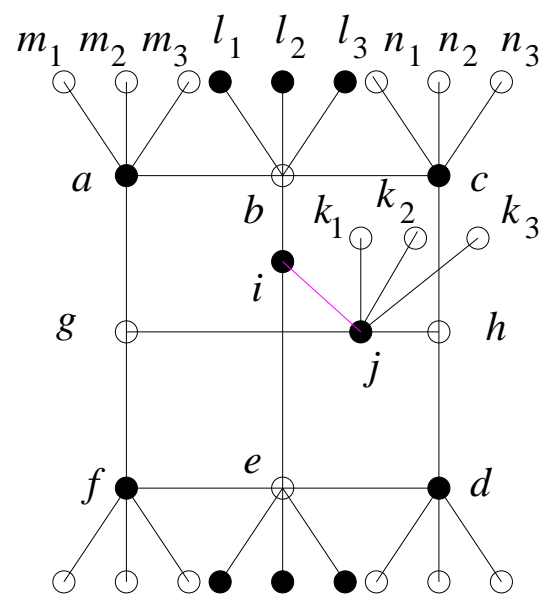

Figure 1.4: The frame graph with 21 vertices attached

Finally, we arrive at our counterexample, which we shall denote by $G_{0}$, by attaching four Heawood triples to six of the seven copies of $K_{1.3}$ via the additional edges shown in Figure 1.6. We define these additional edges as follows. First consider the edges joining the Heawood triple $3 H_{1}$ to the set $V_{21}$. Join vertex $m_{1}$ to vertices 1 in $H_{3}$ and 11 in $H_{1}$; vertex $m_{2}$ to vertices 1 in $H_{2}$ and 11 in $H_{3}$ and vertex $m_{3}$ to vertices 1 in $H_{1}$ and 11 in $H_{2}$. Join vertex $\ell_{1}$ to vertices 4 in $H_{1}$ and 8 in $H_{2}$; vertex $\ell_{2}$ to vertices 4 in $H_{3}$ and 8 in $H_{1}$ and vertex $\ell_{3}$ to vertices 4 in $H_{2}$ and 8 in $H_{3}$.

Now we prescribe the edges joining the Heawood triple $3 H_{2}$ to $V_{21}$. Join vertex $n_{1}$ to vertices 1 in $H_{3}$ and 11 in $H_{1}$; vertex $n_{2}$ to vertices 1 in $H_{2}$ and 11 in $H_{3}$ and vertex $n_{3}$ to vertices 1 in $H_{1}$ and 11 in $H_{2}$. Join vertex $\ell_{1}$ to vertices 4 in $H_{1}$ and 8 in $H_{2}$; vertex $\ell_{2}$ to vertices 4 in $H_{3}$ and 8 in $H_{1}$ and vertex $\ell_{3}$ to vertices 4 in $H_{2}$ and 8 in $H_{3}$. 


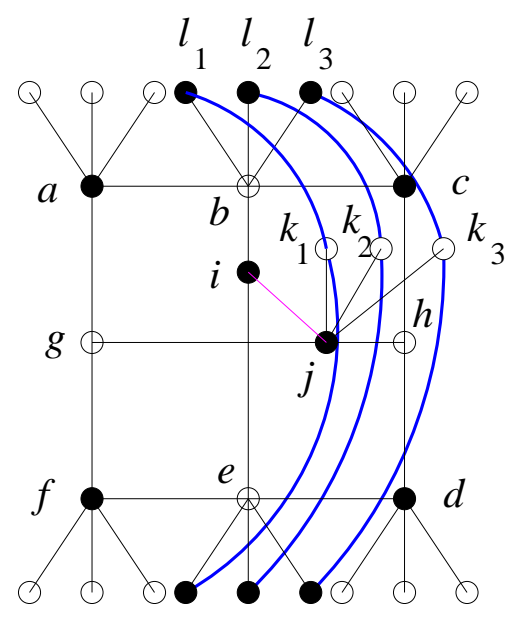

Figure 1.5: The frame graph with 21 vertices attached and six edges added

The nine vertices in $V_{21}$ which lie below the $X Y$-axis are joined to Heawood triples $3 H_{3}$ and $3 H_{4}$ by reflecting in the $X Y$-axis the edges which join $V_{21}$ to $3 H_{1} \cup 3 H_{2}$ already constructed in the preceding two paragraphs.

Note that $G_{0}$ is not bipartite, but if the single edge $i j$ is deleted, the resulting graph is bipartite. The black and white vertices in Figure 1.6 denote the corresponding bipartition of graph $G_{0}-i j$. Note also that the graph $G-i$ is symmetric about the $X Y$-axis.

\section{Properties of the graph $G_{0}$}

Lemma 2.1. The girth of $G_{0}$ is five.

Proof. Easily checked.

Lemma 2.2. Every odd cycle of length greater than 5 in $G_{0}$ has a chord.

Proof. Suppose $Z$ is an odd cycle of length greater than 5 which has no chord. Then $Z$ must use edge $i j$, since $G-i j$ is bipartite.

Moreover, cycle $Z$ cannot contain two vertices from $G-i-j-k_{1}-k_{2}-k_{3}$, one lying above the $X Y$-axis and the other below, because any path between such a pair of vertices which does not contain $i$ and $j$ must contain a neighbor of $j$ and hence $Z$ contains a chord and we have a contradiction. So without loss of generality, let us suppose that the part of $Z$ not containing any of the vertices $i, j, k_{1}, k_{2}$ or $k_{3}$ has no vertex below the $X Y$-axis. Then the edge $b i$ belongs to $Z$ and the next vertex after $i$ and $j$ on $Z$ must be either $g, h$ or one of $k_{1}, k_{2}$ or $k_{3}$. But then $Z$ also contains either $a, c$ or one of $l_{1}, l_{2}$ or $l_{3}$, respectively. Together with vertex $b$, this produces a chord in $Z$ and again we have a contradiction.

Lemma 2.3. The graph $G_{0}$ is internally 4-connected. 


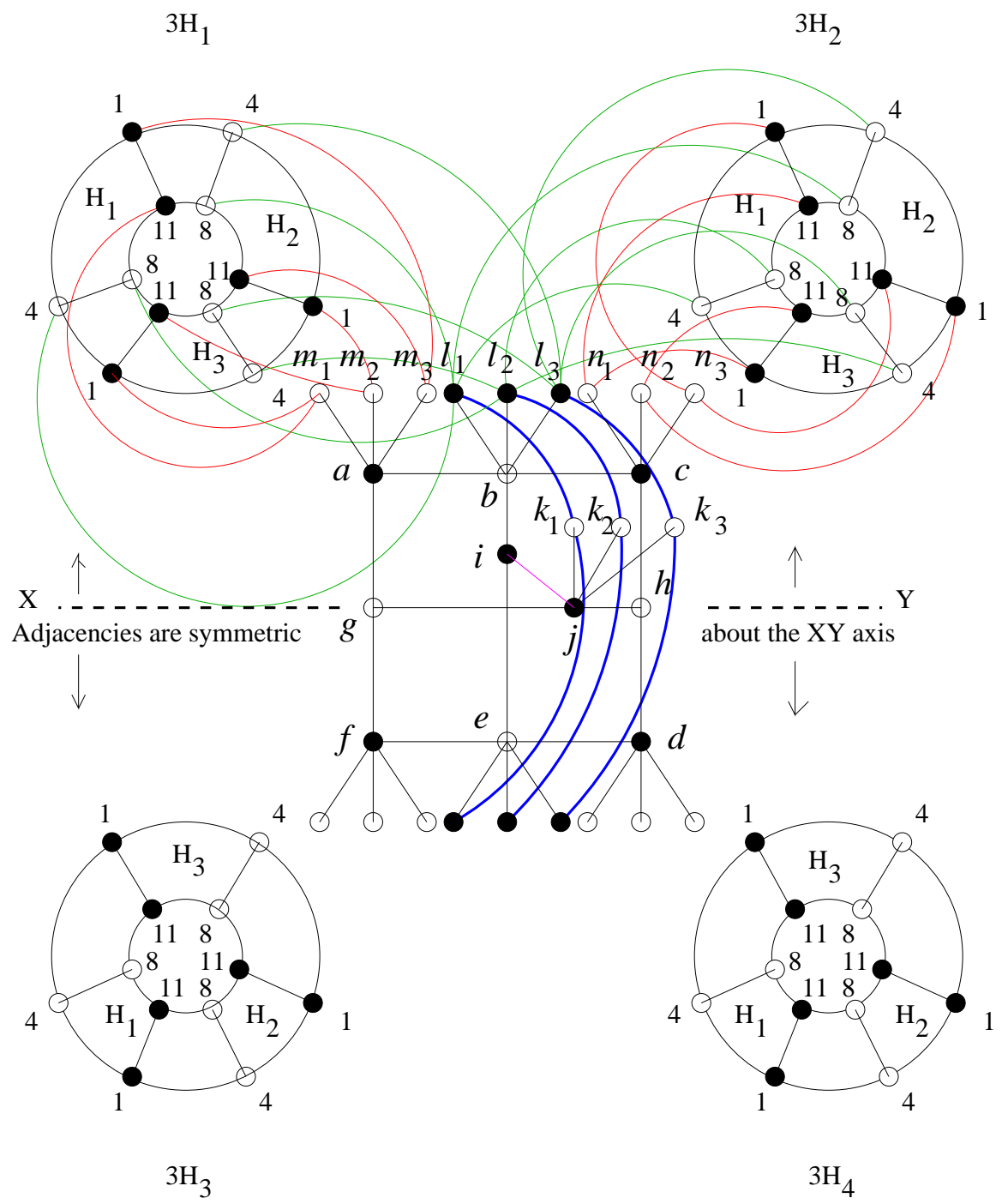

Figure 1.6: The counterexample graph $G_{0}$

Proof. This proof essentially amounts to case checking and we offer one approach to carrying this out. Let the four Heawood triples in $G_{0}$ be labeled $3 H_{i}, 1 \leqslant i \leqslant 4$ as shown in Figure 1.6. By symmetry, without loss of generality, let us focus on the Heawood graph $H_{1}$ belonging to the Heawood triple $3 H_{1}$ and having the vertex labeling shown in Figure 1.6. Claim 2.3.1: $H_{1}$ is internally 4 -connected.

This is easily checked.

Claim 2.3.2: Every edge in $H_{1}$ is incident with four internally disjoint paths ending in vertices $1,4,8$ and 11, respectively. Such a path may consist of a single vertex in the case when the edge is incident with vertices $1,4,8$ and 11 .

This follows from Claim 2.3.1.

Claim 2.3.3: $3 H_{1}$ is internally 4 -connected. 
This too is easy to verify using Claim 2.3.2 and the fact that $H_{1}$ is internally 4connected and that there is a matching of size 4 joining vertices $1,4,8$ and 11 in $H_{1}$ to the rest of $3 H_{1}-V\left(H_{1}\right)$.

Claim 2.3.4: Each edge in $3 H_{1}$ has four internally disjoint paths to every edge in the frame subgraph $\mathcal{F}$. More particularly, there is one such path entering $\mathcal{F}$ at vertex $a$ and three entering at vertices $\ell_{1}, \ell_{2}$ and $\ell_{3}$. Of the latter three paths, one continues to vertex $b$ and the other two use two disjoint edges to some two of $k_{1}, k_{2}$ and $k_{3}$.

Again, this is easily checked.

Henceforth, by way of contradiction, let $S=\left\{s_{1}, s_{2}, s_{3}\right\}$ denote a 3 -cut in $G_{0}$ which is not the neighborhood set of any single vertex.

Claim 2.3.5: $S \nsubseteq V\left(H_{1}\right)$; that is, $\left|S \cap V\left(H_{1}\right)\right| \neq 3$.

Suppose to the contrary, that $S \subseteq V\left(H_{1}\right)$. Let $x$ be any vertex in $V\left(H_{1}\right)-S$ and $x y$ be any edge in $H_{1}-S$ incident with vertex $x$. Note that such an edge $x y$ exists by the definition of $S$. Then $x y$ is incident with four paths going to the vertices $1,4,8$ and 11. Hence there is a path from $x$ to the subgraph $G-S-V\left(H_{1}\right)$. But then $G-S$ is connected, a contradiction.

Claim 2.3.6: $\left|S \cap V\left(H_{1}\right)\right| \neq 2$.

Suppose to the contrary that $S \cap V\left(H_{1}\right)=\left\{s_{1}, s_{2}\right\}$. Since $H_{1}$ is 3-connected, $H_{1}-S$ is connected. Again, any edge $x y$ in $H_{1}$ is incident with four paths to vertices $1,11,4$ and 8 and these paths intersect only at a subset of $\{x, y\}$. But $S=\left\{s_{1}, s_{2}\right\}$ can block at most two of these paths. Now any edge in $H_{1}$ sends at least two internally disjoint paths to two vertices in the set $\{1,4,8,11\}$. Call these two vertices $z_{1}$ and $z_{2}$.

Now let $J$ denote the subgraph of $G_{0}$ induced by $\left(V\left(G_{0}-V\left(H_{1}\right)\right) \cup\{1,4,8,11\}\right.$. Since $J$ is 2-connected, for any vertex $w \in V(J)$ there are two internally disjoint paths from $w$ to $z_{1}$ and $z_{2}$. At most one of these two paths can contain vertex $s_{3}$ and so $G_{0}-S$ is connected, a contradiction.

Claim 2.3.7: $\left|S \cap V\left(H_{1}\right)\right| \neq 1$.

Suppose, to the contrary, that $S \cap V\left(H_{1}\right)=\left\{s_{1}\right\}$. Then both $H_{1}-\left\{s_{1}\right\}$ and $(G-$ $\left.V\left(H_{1}\right)\right)-\left\{s_{2}, s_{3}\right\}$ are connected. By Claims 2.3.3 and 2.3.4, every edge in $G-V\left(H_{1}\right)$ is incident with four internally disjoint paths to vertices $1,4,8$ and 11 respectively in $H_{1}$. Therefore every vertex of $H_{1}-\left\{s_{1}\right\}$ is connected to every vertex in $\left(G-V\left(H_{1}\right)\right)-\left\{s_{2}, s_{3}\right\}$ by a path. Hence $S$ is not a vertex cut and the Claim is proved.

Claim 2.3.8: $\left|S \cap V\left(3 H_{1}\right)\right| \neq 3$.

Claim 2.3.9: $\left|S \cap V\left(3 H_{1}\right)\right| \neq 2$.

The proofs of these two claims are essentially the same as those of Claims 2.3.5 and 2.3.6 together with Claim 2.3.4.

Claim 2.3.10: $\left|S \cap V\left(3 H_{1}\right)\right| \neq 1$. 
Assume, to the contrary, that $S \cap V\left(3 H_{1}\right)=\left\{s_{1}\right\}$. Now $3 H_{1}-\left\{s_{1}\right\}$ is connected (in fact, 2-connected). It is easy to check that there are four vertex-disjoint paths joining $3 H_{1}$ to any of the other three Heawood triples; four vertex-disjoint paths joining $3 H_{1}$ to the set $V_{21}$, and four vertex-disjoint paths joining $3 H_{1}$ to the frame $\mathcal{F}$.

Now by Claims 2.3.8 and 2.3.9, for each of the four Heawood triples $3 H_{i},\left|V\left(3 H_{i}\right) \cap S\right| \leqslant$ 1 and hence $3 H_{i}-S$ is connected. Moreover, then, $\cup_{i=1}^{4} 3 H_{i}-S$ is connected and it follows that $G-S$ is connected, a contradiction.

By symmetry, Claims 2.3.1 through 2.3.10 hold for any other $H_{i}$ and any other $3 H_{i}$, $i=2,3,4$.

So we may now assume that the cutset $S$ is contained in $V_{21} \cup V(\mathcal{F})$.

Now the subgraph spanned by $\cup_{i=1}^{4} V\left(3 H_{i}\right)$ is joined to the frame $\mathcal{F}$ through eighteen of the twenty-one vertices of $V_{21}$. Let us denote by $V_{21}^{a, b, c}$ the subset of $V_{21}$ consisting of the nine vertices collectively adjacent to $\{a, b, c\}$ and by $V_{21}^{d, e, f}$ the subset of $V_{21}$ consisting of the nine vertices collectively adjacent to $\{d, e, f\}$. Then $V_{21}^{a, b, c}$ is joined to $V_{21}^{d, e, f}$ by three vertex-disjoint paths which avoid the frame $\mathcal{F}$. (These paths use the vertices $k_{1}, k_{2}$ and $k_{3}$ shown in Figure 1.6.) Hence altogether there are six vertex-disjoint paths joining $V_{21}^{a, b, c}$ and $V_{21}^{d, e, f}$ in $G_{0}-\cup_{i=1}^{4} V\left(3 H_{i}\right)$.

Claim 2.3.11: $|S \cap V(\mathcal{F})| \neq 3$.

Suppose to the contrary that $|S \cap V(\mathcal{F})|=3$ and hence $S \subseteq V(\mathcal{F})$. Let $K=$ $G_{0}\left[V\left(G_{0}\right)-\{b, e, g, h, i, j\}\right]$ and note that $K$ is connected. Moreover, there are two vertexdisjoint paths $P_{1}$ and $P_{2}$ in $K$, where $P_{1}$ joins vertices $a$ and $d$ and $P_{2}$ joins vertices $c$ and $f$. But then $V(\mathcal{F}) \cup V\left(P_{1}\right) \cup V\left(P_{2}\right)$ induces a subdivision of the Petersen graph in which the only subdivided edges are $a d$ and $c f$. But the Petersen graph is internally 4-connected and both $P_{1}$ and $P_{2}$ are contained in $K$, and hence $S$ cannot be a 3-cut of $G_{0}$ and the Claim is proved.

Claim 2.3.12: $|S \cap V(\mathcal{F})| \neq 2$.

To prove this, let us assume, by way of contradiction, that $|S \cap V(\mathcal{F})|=2$, that $S \cap V(\mathcal{F})=\left\{s_{1}, s_{2}\right\}$ and $S \cap V\left(K^{\prime}\right)=\left\{s_{3}\right\}$, where $K^{\prime}$ is the subgraph spanned by $\left(\cup_{i=1}^{4} V\left(3 H_{i}\right)\right) \cup V_{21}$. It is easy to see that each Heawood triple has three internally disjoint paths to any other Heawood triple which do not use any vertices in $\mathcal{F}$. On the other hand, each vertex in $V_{21}$ has two internally disjoint paths to any of the four Heawood triples where neither of these two paths uses any vertices of $\mathcal{F}$. It follows that $K^{\prime}$ is 2-connected and hence $K^{\prime}-\left\{s_{3}\right\}$ is connected. Claim 2.3.4 shows that each edge in $\mathcal{F}$ has four internally disjoint paths to each of the four Heawood triples.

Hence $\mathcal{F}-\left\{s_{1}, s_{2}\right\}$ is connected. But there is a path joining $\mathcal{F}-\left\{s_{1}, s_{2}\right\}$ and $K^{\prime}$ and it then follows that $G_{0}-S$ is connected, a contradiction.

Claim 2.3.13: $|S \cap V(\mathcal{F})| \neq 1$.

Again, by way of contradiction, assume that $|S \cap V(\mathcal{F})|=1$ and that $S \cap V(\mathcal{F})=\left\{s_{1}\right\}$. By Claims 2,3,8, 2.3.9, and 2.3.10, $S \cap\left(\cup_{i=1}^{4} V\left(3 H_{i}\right)\right)=\emptyset$ for $i=1, \ldots, 4$. Therefore, $\left\{s_{2}, s_{3}\right\} \subseteq V_{21}$. Clearly, 


$$
\mathcal{F}-\left\{s_{1}\right\} \text { is connected. }
$$

We now claim that $K^{\prime}-\left\{s_{2}, s_{3}\right\}$ is connected.

Suppose to the contrary that $\left\{s_{2}, s_{3}\right\}$ separates $K^{\prime}$. Graph $K^{\prime}$ is a subdivision of a 3 -connected graph in which the only degree 2 vertices lie among the fifteen white vertices of $V_{21}$. (Cf. Figure 1.6.) All possible 2-vertex cuts of $K^{\prime}$ consist of neighbor sets of the fifteen degree 2 vertices of $K^{\prime}$. Twelve of these 2-vertex cuts are contained in $\cup_{i=1}^{4} V\left(3 H_{i}\right)$, while the remaining three 2-vertex cuts consist of neighbors in $K^{\prime}$ of vertices in the set $\{h, j, k\}$. But since $S \cap\left(\cup_{i=1}^{4} V\left(3 H_{i}\right)\right)=\emptyset$, the set $\left\{s_{2}, s_{3}\right\}$ must be the neighbor set of one of the vertices in $\{h, j, k\}$. It is easy to check that in this case,

$$
K^{\prime}-\left\{s_{2}, s_{3}\right\} \text { is connected. }
$$

But now by Claims 2.3.4, $\left.{ }^{* *}\right)$ and $\left({ }^{* *}\right)$, it follows that $G_{0}-S$ is connected, a contradiction. This proves Claim 2.3.13.

But then it must be the case that $S \cap V(\mathcal{F})=\emptyset$ and hence that $S \subseteq V_{21}$. However, the reader can easily check that no subset of $V_{21}$ of size 3 disconnects $G_{0}$.

\section{Concluding Remarks}

We would like to conclude by raising some questions and posing four conjectures for future study. Let us define the class $\mathcal{G}$ to be the class of those graphs $G$ which are (a) 3 -connected, (b) internally 4-connected, (c) of girth 5 and (d) such that every odd cycle of length greater than 5 has a chord.

We adopt two somewhat different approaches.

(1) Suppose $G \in \mathcal{G}$ and $C$ is a girth cycle in $G$. During our approach to Robertson's Conjecture, we noted that the subgraph induced by $C \cup N(C)$ must be very close in structure to certain subgraphs of the Petersen graph. Let $\mathcal{F}$ be the frame graph introduced in Section 1 (cf. Figure 1.3) which consists of the Petersen graph with two disjoint edges at distance 2 deleted. We propose the following conjectures.

Conjecture 3.1. Suppose $G \in \mathcal{G}$ and $C$ is any 5-cycle in $G$. Then $G$ contains a subgraph which, in turn, contains $C$ and is isomorphic to $\mathcal{F}$.

Conjecture 3.2. Suppose $G \in \mathcal{G}$ and let $C$ be any 5 -cycle of $G$. Then $G$ contains a subgraph that contains $C$ and is isomorphic to the Petersen graph with two edges at distance 2 subdivided.

If both conjectures are true, it would indicate that in every counterexample graph $G$ and every girth cycle $C$ in $G$, there is a subgraph $H$ of $G$ which contains $C$ and that is "close to" the Petersen graph. 
We understand that Robertson proposed his conjecture during his work on the Perfect Graph Conjecture. The Strong Perfect Graph Conjecture (recently proved by Chudnovsky, Robertson, Seymour and Thomas [1]) states that a graph $H$ is is perfect if and only if $H$ contains neither an odd hole nor an odd anti-hole. (An odd hole is an induced subgraph isomorphic to an odd cycle and an odd anti-hole is the complement of an odd hole.)

For graphs in $\mathcal{G}$, the conditions that the girth be 5 and that every odd cycle of length at least 5 has a chord is equivalent to saying that the only odd holes in $G$ must be of size 5. In contrast to perfect graphs which do not have any odd holes, graphs in $\mathcal{G}$ are allowed odd holes of size 5 only. As viewed from this perspective, we see again that the structure of graphs in the class $\mathcal{G}$ may be very close to that of perfect graphs.

(2) Secondly, we point out that the counterexample constructed in this paper is just one edge away from being a bipartite graph; i.e., the graph $G_{0}-i j$ is bipartite. We believe that any counterexample to Robertson's Conjecture must have a similar structure and hence that the graphs in $\mathcal{G}$ are either the Petersen graph or are "close" to being bipartite. While bipartite graphs are perfect, it is also suggested that graphs in class $\mathcal{G}$, if not the Petersen graph, should be "close" to being perfect.

Question 3.3. How close to being perfect are the graphs in class $\mathcal{G}$, other than the Petersen graph?

Certainly the term "close" is not well-defined in the preceding question.

Perfect graphs were originally defined in terms of graph coloring. That is, a graph $G$ is perfect if the chromatic number of $G$ is equal to the size of a largest clique in $G$.

We close with the following closely related question.

Question 3.4. Must a graph in class $\mathcal{G}$ have small chromatic number? Bounded chromatic number? (We conjecture that the chromatic number of graphs in $\mathcal{G}$ is 3 .)

\section{References}

[1] M. Chudnovsky, N. Robertson, P. Seymour and R. Thomas, The strong perfect graph theorem, Ann. of Math. 164 (2006) 51-229.

[2] D. Nelson, M. Plummer, N. Robertson and X. Zha, On a conjecture concerning the Petersen graph, Electron. J. Combin. 18(1) (2011), P20, 37pp.

[3] N. Robertson, personal communication to second author.

[4] http://en.wikipedia.org/wiki/Heawood_graph 